\title{
Lesson of the month 2: A rare presentation of stroke: diagnosis made on magnetic resonance imaging
}

\author{
Authors: Danielle Berenson, ${ }^{\mathrm{A}}$ Luke Nuttall, ${ }^{\mathrm{B}}$ Eluzai Hakim ${ }^{\mathrm{C}}$ and Khaled Abdel-Aziz ${ }^{\mathrm{D}}$
}

\begin{abstract}
Bilateral thalamic infarcts are uncommon posterior circulation strokes. The artery of Percheron (AOP) is a rare anatomical variant involving a singular arterial supply to both thalami and occlusion leads to bilateral thalamic infarction.

We report the case of a 71-year-old man who presented with decreased consciousness (fluctuating Glasgow Coma Scale score of 5-7). He had a background of atrial fibrillation and was anticoagulated with dabigatran, a novel oral anticoagulant. Computed tomography (CT) scan showed a mildly reduced attenuation in the region of the left thamalus. Subsequent diffusion-weighted magnetic resonance imaging (MRI) showed acute brainstem infarction, extending into the thalamus bilaterally, likely due to AOP occlusion. Bilateral thalamic infarcts due to AOP occlusion may not be recognised on initial CT scan and are more readily seen using diffusionweighted MRI, which is the most beneficial imaging modality to aid in early diagnosis and treatment.
\end{abstract}

KEYWORDS: Artery of Percheron, bilateral thalamic infarcts, novel oral anticoagulants, diffusion-weighted imaging, atrial fibrillation

\section{Case description}

A 71-year-old man with a background of hypertension and atrial fibrillation (AF) was admitted to the Emergency Department after being found unresponsive by his family, having previously been well that day. He had a fluctuating Glasgow Coma Score between 5-7 on admission and was therefore immediately transferred to the intensive care unit, where he was intubated.

\section{Investigations}

A computed tomography (CT) head scan showed low attenuation in the left thalamic region.

Authors: A senior house officer, general internal medicine, Ashford and St Peter's NHS Trust, Chertsey, UK; ${ }^{\text {B }}$ foundation year 1 doctor, general internal medicine, Ashford and St Peter's NHS Trust, Chertsey, UK; ' Consultant stroke physician, Ashford and St Peter's NHS Trust, Chertsey, UK; ${ }^{D}$ consultant neurologist, Ashford and St Peter's NHS Trust, Chertsey, UK
Electroencephalography showed an abnormally slow trace, potentially due to sedation or mild encephalopathy.

Subsequent magnetic resonance imaging (MRI) of the brain showed acute infarction in the brainstem extending into the thalamus bilaterally, likely due to obstruction of the artery of Percheron (AOP) (see Fig 1a, b and c).

\section{Subsequent events}

The patient had been on dabigatran, a novel oral anticoagulant for stroke prophylaxis due to his history of AF. Aspirin $300 \mathrm{mg}$ once daily was started via nasogastric tube and after 14 days, clopidogrel $75 \mathrm{mg}$ once daily was substituted as per standard treatment protocol for ischaemic stroke.

He subsequently developed fast AF, for which he was loaded on digoxin and amiodarone, and seizure activity, requiring levetiracetam, sodium valproate and midazolam. Numerous extubation attempts failed and a tracheostomy tube was sited. He developed Streptococcus cristatus septicaemia, recurrent ventilator-associated pneumonias, cellulitis around the tracheostomy site and a third-nerve palsy secondary to the infarction in the dorsal midbrain, where the oculomotor nucleus lies. A percutaneous endoscopic gastrostomy was sited for feeding and medication administration.

After initial overall improvement, the patient developed right facial and arm tics and became completely unresponsive. Bedside electroencephalography showed status epilepticus and a midazolam infusion, along with clonazepam, was added in to his anti-seizure management. Thiopentone infusion was started; however, electroencephalogram showed a worsening rhythm so this was weaned. Phenobarbital was trialled and the possibility of using ketamine or magnesium was raised for the management of super-refractory status epilepticus.

After discussing this and the poor prognosis with the family, a palliative approach was decided upon, followed by withdrawal of treatment. The patient passed away peacefully.

\section{Discussion}

Thalamic arterial infarcts can be classified according to their vascular territories, each of which supplies particular clusters of nuclei. $^{1}$

Paramedian arterial infarcts, involving the paraventricular and intralaminar nuclei, cause neuropsychological disturbance, impaired learning, language deficits, memory disturbance, 
Fig 1. MRIs depicting sites of acute infarction. (a) T2 FLAIR showing bilateral paramedian thalamic infarcts. (b,c) Diffusionweighted imaging (DWI) and apparent diffusion coefficient (ADC) map showing restricted diffusion within the paramedian thalamic nuclei.

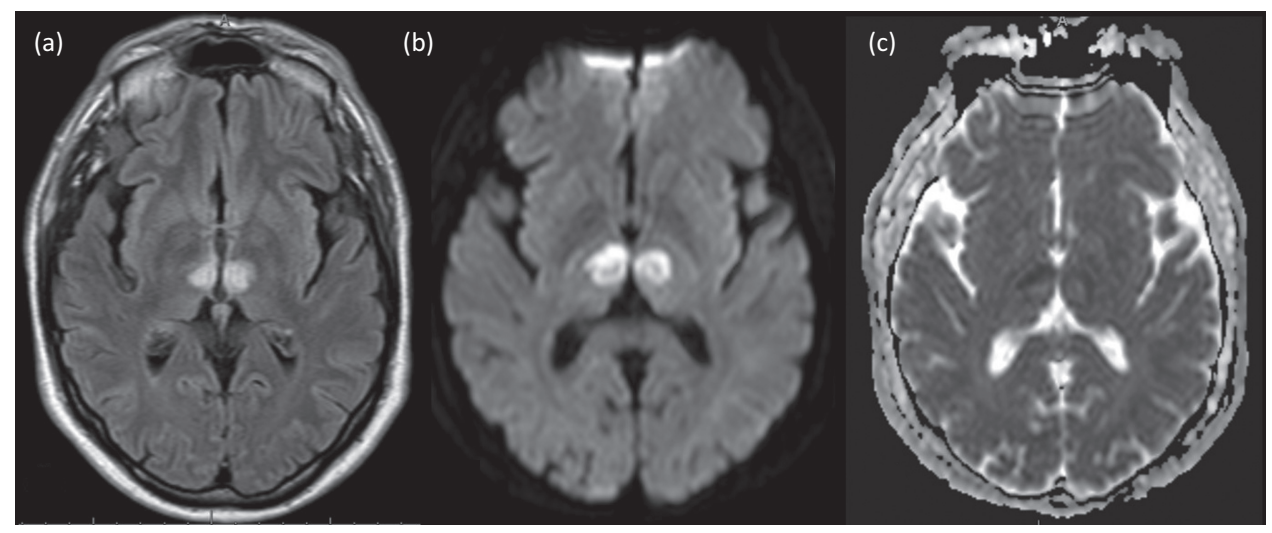

vertical gaze palsy, tremor and decreased arousal. Coma can occur if the infarct is bilateral. Bithalamic ischaemic strokes may raise suspicion of the presence of the AOP, which is a single paramedian arterial trunk supplying both thalamic territories. ${ }^{1}$ This anatomical variant is often notable on MRI with diffusionweighted imaging (DWI), as was the case for this patient. The patient did not have conventional angiography, but would have had he survived to confirm AOP presence. However, occlusion may lead to lack of visualisation of the AOP on angiography, even if it is present. ${ }^{2}$

Inferolateral arterial infarcts, often involving the ventroposterior complex and ventral lateral nucleus, can cause personality change, hemiparesis, hemisensory loss and pain syndromes. Posterior choroidal arterial infarcts, often involving the medial and lateral geniculate nuclei can lead to weakness and sensory loss, audiovisual defects, tremors, and memory and language impairment. Tuberothalamic arterial infarcts, often involving reticular and intralaminar nuclei, can cause reduced arousal and orientation, memory and learning impairment and personality change, including apathy. ${ }^{1}$

\section{Differential diagnosis}

The differential diagnosis of thalamic infarcts also includes central venous sinus thrombosis (CVT). Presentation often involves rapidly reducing consciousness, headache, hemiparesis and aphasia. ${ }^{3}$ Predisposing factors include trauma, pregnancy, oral contraceptive pill, infection, antithrombin III deficiency, proteins $\mathrm{C}$ and $\mathrm{S}$ deficiency, antiphospholipid syndrome and cancer. ${ }^{4}$ Thalamic hypoattenuation, haemorrhagic transformation and hydrocephalus secondary to venous oedema may be seen on $\mathrm{CT}^{3}$ Arterial infarcts produce similar $\mathrm{CT}$ findings and are differentiated from venous infarcts using DWI. Arterial infarcts with oedema cause restricted diffusion, as seen in this case, whereas venous infarcts with oedema causes increased diffusion. ${ }^{3}$

\section{Management}

Guidelines for management of CVT involve management of symptoms, addressing the underlying condition and complication prevention. This encompasses initiating anticoagulation with heparin or low-molecular-weight heparin and subsequently switching to oral anticoagulation if the patient remains stable or improves neurologically. ${ }^{4}$ This aims to prevent deep vein thrombosis (DVT) and pulmonary embolism (PE), to facilitate recanalisation and halt thrombus growth. Anticoagulants should be continued for up to 12 months if the CVT is an isolated event, or indefinitely for those with recurrent CVT or predisposing conditions such as antiphospholipid syndrome. ${ }^{4}$ Thrombectomy, thrombolysis or surgical management, such as decompressive hemicraniectomy if haemorrhage is demonstrated, may also be considered. ${ }^{4}$ It is recommended that antiepileptic medications are started after a single seizure occurs. ${ }^{4}$

Management of arterial thrombosis includes consideration of thrombolysis with within 4.5 hours of symptom onset if intracranial haemorrhage has been ruled out. ${ }^{5}$ Aspirin with proton pump inhibitor cover is recommended, with subsequent long-term antithrombotic cover using agents such as clopidogre or dipyridamole. If there is concomitant symptomatic DVT/PE, anticoagulation is advised in preference to antiplatelet therapy if there are no contraindications. ${ }^{5}$ Anticoagulation, including the use of direct oral anticoagulants (DOACs) such as rivaroxaban, is recommended for stroke prevention in patients with $\mathrm{AF}^{5}{ }^{5}$ Antihypertensives are recommended if a hypertensive emergency occurs, such as hypertensive encephalopathy or aortic dissection. ${ }^{5}$ Lifestyle changes such as stopping smoking and low-cholesterol diets should also be advised.

\section{Learning points}

> Artery of Percheron (AOP) presence, although rare, should be considered as a differential diagnosis in sudden-onset reduced responsiveness, speech disturbance or gaze palsies.

> Bilateral thalamic infarction secondary to AOP occlusion may not be recognised on initial $\mathrm{CT}$ scan.

> The imaging modality of choice for recognition of bilateral thalamic infarction secondary to AOP occlusion is diffusionweighted MRI imaging.

> The use of diffusion-weighted MRI may lead to earlier AOP occlusion diagnosis and management.

\section{Consent}

Written consent was obtained from the next of kin to submit this article for publishing. 


\section{Author contributions}

Dr Berenson led the writing of the manuscript for this case report and Dr Nuttall aided in case history collection. Dr Hakim selected the case initially and both he and Dr Abdel-Aziz were both involved in the management of this patient and revising the manuscript for intellectual content.

\section{Acknowledgements}

Many thanks to the Radiology Department at St Peter's Hospital who aided in sourcing the image for this case report.

\section{References}

1 Schmahmann JD. Vascular syndromes of the thalamus. Stroke 2003;34:2264-78.

2 Matheus MG, Castillo M. Imaging of acute bilateral paramedian thalamic and mesencephalic infarcts. AJNR Am J Neuroradiol 2003;24:2005-8.
3 Hermann KA, Sporer B, Yousry TA. Thrombosis of the internal cerebral vein associated with transient unilateral thalamic edema: a case report and review of the literature. AJNR Am J Neuroradiol 2004:25:1351-5.

4 Saposnik G, Barinagarrementeria F, Brown R et al. Diagnosis and management of cerebral venous thrombosis. Stroke 2011;42:1158-92.

5 National Institute for Health and Care Excellence. Stroke and transient ischaemic attack in over 16s: diagnosis and initial man agement. NICE, 2008 https://www.nice.org.uk/guidance/cg68 [Accessed 22 March 2017].

Address for correspondence: C/O Dr Eluzai Hakim, Cedar Ward, St. Peter's Hospital, Guildford Road, Chertsey KT16 OPZ, United Kingdom.

Email: danielle.berenson@doctors.org.uk
Order by phone or online

Tel +44 (0)20 30751358

or visit www.rcplondon.ac.uk/shop

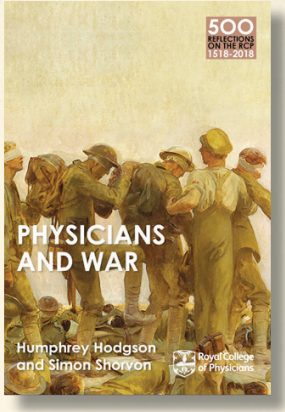

Price: $€ 12$

(Postage and packaging not included)

$10 \%$ discount for fellows and members,

$15 \%$ discount for foundation doctors and students 